\title{
The double copy: Bremsstrahlung and accelerating black holes
}

\author{
Andrés Luna, ${ }^{a}$ Ricardo Monteiro, ${ }^{b}$ Isobel Nicholson, ${ }^{c}$ Donal $0^{\prime}$ Connell ${ }^{c, d}$ and \\ Chris D. White ${ }^{a}$ \\ a School of Physics and Astronomy, University of Glasgow, \\ Glasgow G12 8QQ, Scotland, U.K. \\ ${ }^{b}$ Theoretical Physics Department, CERN, \\ Geneva, Switzerland \\ ${ }^{c}$ Higgs Centre for Theoretical Physics, School of Physics and Astronomy, \\ The University of Edinburgh, \\ Edinburgh EH9 3JZ, Scotland, U.K. \\ ${ }^{d}$ Kavli Institute for Theoretical Physics, University of California, \\ Santa Barbara, CA 93106-4030 U.S.A. \\ E-mail: a.luna-godoy.1@research.gla.ac.uk, ricardo.monteiro@cern.ch, \\ i.nicholson@sms.ed.ac.uk, donal@staffmail.ed.ac.uk, \\ Christopher.White@glasgow.ac.uk
}

ABSTRACT: Advances in our understanding of perturbation theory suggest the existence of a correspondence between classical general relativity and Yang-Mills theory. A concrete example of this correspondence, which is known as the double copy, was recently introduced for the case of stationary Kerr-Schild spacetimes. Building on this foundation, we examine the simple time-dependent case of an accelerating, radiating point source. The gravitational solution, which generalises the Schwarzschild solution, includes a non-trivial stress-energy tensor. This stress-energy tensor corresponds to a gauge theoretic current in the double copy. We interpret both of these sources as representing the radiative part of the field. Furthermore, in the simple example of Bremsstrahlung, we determine a scattering amplitude describing the radiation, maintaining the double copy throughout. Our results provide the strongest evidence yet that the classical double copy is directly related to the BCJ double copy for scattering amplitudes.

KeYwords: Scattering Amplitudes, Gauge Symmetry

ArXiv EPrint: 1603.05737 


\section{Contents}

1 Introduction 1

2 Review of the Kerr-Schild double copy $\quad 3$

3 Kerr-Schild description of an accelerating point particle 5

3.1 Gravity solution 5

$\begin{array}{ll}3.2 & \text { Single copy }\end{array}$

4 From Kerr-Schild sources to amplitudes $\quad 9$

$\begin{array}{ll}4.1 \text { Gauge theory } & 10\end{array}$

$\begin{array}{ll}4.2 \text { Gravity } & 11\end{array}$

5 Gravitational energy conditions $\quad 13$

$\begin{array}{lll}6 & \text { Discussion } & 14\end{array}$

$\begin{array}{ll}\text { A Fourier transform of source terms } & 16\end{array}$

\section{Introduction}

Our most refined understanding of nature is founded on two major theoretical frameworks: general relativity and Yang-Mills theory. There is much in common between these two: local symmetries play an important role in their structure; there are simple action principles for both theories; the geometry of fibre bundles is common to the physical interpretation of the theories. But at the perturbative level, general relativity seems to be a vastly different creature to Yang-Mills theory. Indeed, the Einstein-Hilbert Lagrangian, when expanded in deviations of the spacetime metric from some fiducial metric (such as the Minkowski metric) contains terms with arbitrarily many powers of the deviations. This is in stark contrast to the Yang-Mills Lagrangian, which contains at most fourth order terms in perturbation theory.

From this perturbative point of view, it is therefore remarkable that Kawai, Lewellen and Tye (KLT) found [1] that every tree scattering amplitude in general relativity can be expressed as a sum over products of two colour-stripped Yang-Mills scattering amplitudes. Therefore the KLT relations and the Yang-Mills Lagrangian together can be used to reconstruct the Lagrangian of general relativity [2]. This suggests that there may be a KLT-like map between solutions of general relativity and solutions of Yang-Mills theory.

More recently, the perturbative relationship between gauge and gravity theories has been formulated in a particularly suggestive manner by Bern, Carrasco and Johansson (BCJ) [3-5]. BCJ found that gravity $n$-point amplitudes can be obtained from $n$-point 
gauge theory counterparts at the level of diagrams. Specifically, the BCJ prescription is simply to replace the colour factor of each diagram by an additional copy of the diagram's kinematic numerator. This replacement must be performed in a particular representation of the amplitude, where the kinematic numerators satisfy the algebraic properties of the corresponding colour factor. In particular, the kinematic factors must satisfy the same Jacobi identities and antisymmetry properties as the colour factors. For this reason, the BCJ representation of the kinematic numerators is known as a colour-dual representation. The procedure of replacing colour factors in gauge theory scattering amplitudes with another copy of the kinematic numerator is known as the double copy, since it represents gravity scattering amplitudes as two copies of Yang-Mills scattering amplitudes.

The validity of the BCJ double copy and the existence of colour-dual numerators has been proven at tree-level [5-13] (where it is equivalent to the KLT relations [1]). One very exciting feature of the BCJ procedure is that it admits a simple extension to loop diagrams in the quantum theory [4]. This extension remains conjectural, but it has been verified in highly nontrivial examples at multiloop level [4, 14-35]. All-order evidence can be obtained in special kinematic regimes [17, 36-39], but a full proof of the correspondence has to date been missing (see, however, refs. [40-54] for related studies).

Motivated by this progress, a double copy for classical field solutions (which we will refer to as the classical double copy) has been proposed [55]. This classical double copy is similar in structure to the BCJ double copy for scattering amplitudes: in both cases, the tensor structure of gravity is constructed from two copies of the vector structure of gauge theory. In addition, scalar propagators are present in both cases; these scalars are exactly the same in gauge and gravitational processes. However, the classical double copy [55] is only understood at present for the special class of Kerr-Schild solutions in general relativity. This reflects the particularly simple structure of Kerr-Schild metrics: the Kerr-Schild ansatz has the remarkable property that the Einstein equations exactly linearise. Therefore we can anticipate that any Yang-Mills solution related to a Kerr-Schild spacetime must be particularly simple. Indeed, the authors of [55] showed that any stationary Kerr-Schild solution has a well-defined single copy that satisfies the Yang-Mills equations, which also take the linearised form. While the structure of the classical double copy is very reminiscent of the BCJ double copy, so far no precise link has been made between the two. One aim of the present article is to provide such a link.

Although the classical double copy is only understood for a restricted class of solutions, many of these are familiar. For example, the Schwarzschild and Kerr black holes are members of this class; in higher dimensions, the Myers-Perry black holes are included [55]. The relationship between classical solutions holds for all stationary Kerr-Schild solutions, but other Kerr-Schild solutions are known to have appropriate single copies. A particularly striking example is the shockwave in gravity and gauge theory; the double copy of this pair of solutions was pointed out by Saotome and Akhoury [36]. In further work, the classical double copy has been extended [56] to the Taub-NUT solution $[57,58]$, which has a double Kerr-Schild form and whose single copy is a dyon in gauge theory.

Despite this success, Kerr-Schild solutions are very special and do not easily describe physical systems which seem very natural from the point of view of the double copy for 
scattering amplitudes. For example, there is no two-form field or dilaton on the gravity side; there are no non-abelian features on the gauge theory side; the status of the sources must be better understood. In cases where the sources are point particle-like, the classical double copy relates the gauge theory current density to the gravity energy-momentum tensor in a natural way $[55,56]$. For extended sources, extra pressure terms on the gravity side are needed to stabilise the matter distribution. Furthermore, reference [59] pointed out that in certain gravity solutions the energy-momentum tensor does not satisfy the weak and/or strong energy conditions of general relativity.

The aim of this paper is to extend the classical double copy of refs. $[55,56]$ by considering one of the simplest situations involving explicit time dependence, namely that of an arbitrarily accelerating, radiating point source. We will see that this situation can indeed be interpreted in the Kerr-Schild language, subject to the introduction of additional source terms for which we provide a clear interpretation. One important fact which will emerge is that these sources themselves have a double copy structure. We will demonstrate that the sources can be related directly to scattering amplitudes, maintaining the double copy throughout. This provides a direct link between the classical double copy and the BCJ procedure for amplitudes, strongly bolstering the argument that these double copies are the same. The gravitational solution of interest to us is a time-dependent generalisation of the Schwarzschild solution; we will see that this gravitational system is a precise double copy of an accelerating point particle. Since there is a double copy of the sources, and these describe the radiation fields, we learn that the gravitational radiation emitted by a black hole which undergoes a short period of acceleration is a precise double copy of electromagnetic Bremsstrahlung.

The structure of our paper is as follows. In section 2, we briefly review the Kerr-Schild double copy. In section 3, we present a known Kerr-Schild solution for an accelerating particle, before examining its single copy. We will find that additional source terms appear in the gauge and gravity field equations, and in section 4 we relate these to scattering amplitudes describing radiation, by considering the example of Bremsstrahlung. In section 5, we examine the well-known energy conditions of GR for the solutions under study. Finally, we discuss our results and conclude in section 6 . Technical details are contained in an appendix.

\section{Review of the Kerr-Schild double copy}

Let us begin with a brief review of the Kerr-Schild double copy, originally proposed in $[55,56]$. We define the graviton field via

$$
g_{\mu \nu}=\bar{g}_{\mu \nu}+\kappa h_{\mu \nu}, \quad \kappa=\sqrt{16 \pi G_{\mathrm{N}}}
$$

where $G_{\mathrm{N}}$ is Newton's constant, and $\bar{g}_{\mu \nu}$ is a background metric, which, for the purposes of the present paper, we will take to be the Minkowski metric. ${ }^{1}$ There is a special class of Kerr-Schild solutions of the Einstein equations, in which the graviton has the form

$$
h_{\mu \nu}=-\frac{\kappa}{2} \phi k_{\mu} k_{\nu}
$$

\footnotetext{
${ }^{1}$ We choose to work with a negative signature metric $\eta=\operatorname{diag}(1,-1,-1,-1)$.
} 
consisting of a scalar function $\phi$ multiplying the outer product of a vector $k_{\mu}$ with itself. We have inserted a negative sign in this definition for later convenience. The vector $k_{\mu}$ must be null and geodesic with respect to the background:

$$
\bar{g}_{\mu \nu} k^{\mu} k^{\nu}=0, \quad(k \cdot D) k=0,
$$

where $D^{\mu}$ is the covariant derivative with respect to the background metric. It follows that $k_{\mu}$ is also null and geodesic with respect to the metric $g_{\mu \nu}$. These solutions have the remarkable property that the Ricci tensor with mixed upstairs / downstairs indices is linear in the graviton. More specifically, one has

$$
R_{\nu}^{\mu}=\bar{R}_{\nu}^{\mu}-\kappa\left[h^{\mu}{ }_{\rho} \bar{R}_{\nu}^{\rho}-\frac{1}{2} D_{\rho}\left(D_{\nu} h^{\mu \rho}+D^{\mu} h^{\rho}{ }_{\nu}-D^{\rho} h^{\mu}{ }_{\nu}\right)\right]
$$

where $\bar{R}_{\mu \nu}$ is the Ricci tensor associated with $\bar{g}_{\mu \nu}$, and we have used the fact that $h^{\mu}{ }_{\mu}=0$. It follows that the Einstein equations themselves linearise. Furthermore, ref. [55] showed that for every stationary Kerr-Schild solution (i.e. where neither $\phi$ nor $k^{\mu}$ has explicit time dependence), the gauge field

$$
A_{\mu}^{a}=c^{a} \phi k^{\mu}
$$

for a constant colour vector $c^{a}$, solves the Yang-Mills equations. Analogously to the gravitational case, these equations take a linearised form due to the trivial colour dependence of the solution. We then refer to such a gauge field as the single copy of the graviton $h_{\mu \nu}$, since it involves only one factor of the Kerr-Schild vector $k_{\mu}$ rather than two. Note that the scalar field $\phi$ is left untouched by this procedure. This was motivated in ref. [55] by taking the zeroth copy of eq. (2.5) (i.e. stripping off the remaining $k^{\mu}$ factor), which leaves the scalar field itself. The zeroth copy of a Yang-Mills theory is a biadjoint scalar field theory, and the field equation linearises for the scalar field obtained from eq. (2.5). The scalar function $\phi$ then corresponds to a propagator, and is analogous to the untouched denominators (themselves scalar propagators) in the BCJ double copy for scattering amplitudes.

Source terms for the biadjoint, gauge and gravity theories also match up in a natural way in the Kerr-Schild double copy. Pointlike sources in a gauge theory map to point particles in gravity, where electric and (monopole) magnetic charge are replaced by mass and NUT charge respectively [56]. Extended source distributions (such as that for the Kerr black hole considered in ref. [55]) lead to additional pressure terms in the gravity theory, which are needed to stabilise the source distribution so as to be consistent with a stationary solution. Conceptual questions relating to extended source distributions have been further considered in ref. [59], regarding the well-known energy conditions of general relativity. In this work, we will consider point-like objects throughout, and therefore issues relating to extended source distributions will not trouble us. Nevertheless we will discuss the energy conditions in section 5 below.

Let us emphasise that the Kerr-Schild double copy cannot be the most general relationship between solutions in gauge and gravity theories. Indeed, the field one obtains upon taking the outer product of $k^{\mu}$ with itself is manifestly symmetric. Moreover, the null condition on $k^{\mu}$ means that the trace of the field vanishes. Hence, the Kerr-Schild double 
copy is unable to describe situations in which a two-form and / or dilaton are active in the gravity theory. This contrasts sharply with the double copy procedure for scattering amplitudes, which easily incorporates these fields. Furthermore, Yang-Mills amplitudes only obey the double copy when written in BCJ dual form, meaning that certain Jacobi relations are satisfied by the kinematic numerator functions [3-5]. It is not known what the analogue of this property is in the classical double copy procedure. All of these considerations suggest that the Kerr-Schild story forms part of a larger picture, and in order to explore this it is instructive to seek well-defined generalisations of the results of refs. $[55,56]$.

\section{Kerr-Schild description of an accelerating point particle}

In this article, we will go beyond previous work on the Kerr-Schild double copy $[55,56]$ by considering an accelerating point particle. This is a particularly attractive case, because an accelerating point particle must radiate, so we may hope to make direct contact between the double copy for scattering amplitudes and for Kerr-Schild backgrounds. We first describe a well-known Kerr-Schild spacetime containing an accelerating point particle, before constructing the associated single-copy gauge theoretic solution. We find that the physics of the single copy is particularly clear, allowing a refined understanding of the gravitational system. We will build on this understanding in section 4 to construct a double copy pair of scattering amplitudes from our pair of Kerr-Schild solutions in gauge theory and gravity in a manner that preserves the double copy throughout.

\subsection{Gravity solution}

Consider a particle of mass $M$ following an arbitrary timelike worldline $y(\tau)$, parameterised by its proper time $\tau$ so that the proper velocity of the particle is the tangent to the curve

$$
\lambda^{\mu}=\frac{d y^{\mu}}{d \tau} .
$$

An exact Kerr-Schild spacetime containing this massive accelerating particle is known, though the spacetime contains an additional stress-energy tensor; we will understand the physical role of this stress-energy tensor below. A useful geometric interpretation of the null vector $k_{\mu}$ appearing in the solution has been given in refs. [60-62] (see ref. [63] for a review), as follows. Given an arbitrary point $y^{\mu}(\tau)$ on the particle worldline, one may draw a light cone as shown in figure 1 . At all points $x^{\mu}$ along the light-cone, one may then define the null vector

$$
k^{\mu}(x)=\left.\frac{(x-y(\tau))^{\mu}}{r}\right|_{\mathrm{ret}}, \quad r=\left.\lambda \cdot(x-y)\right|_{\mathrm{ret}},
$$

where the instruction ret indicates that $y$ and $\lambda$ should be evaluated at the retarded time $\tau_{\text {ret }}$, i.e. the value of $\tau$ at which a past light cone from $x^{\mu}$ intersects the worldline. Calculations are facilitated by noting that:

$$
\begin{aligned}
\partial_{\mu} k_{\nu} & =\partial_{\nu} k_{\mu}=\frac{1}{r}\left(\eta_{\mu \nu}-\lambda_{\mu} k_{\nu}-k_{\mu} \lambda_{\nu}-k_{\mu} k_{\nu}(-1+r k \cdot \dot{\lambda})\right), \\
\partial_{\mu} r & =\lambda_{\mu}+k_{\mu}(-1+r k \cdot \dot{\lambda}),
\end{aligned}
$$

where dots denote differentiation with respect to the proper time $\tau$. 


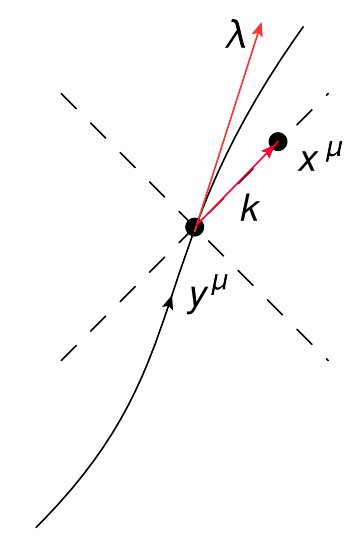

Figure 1. Geometric interpretation of the Kerr-Schild solution for an accelerated particle.

The Kerr-Schild metric associated with this particle is

$$
g_{\mu \nu}=\eta_{\mu \nu}-\frac{\kappa^{2}}{2} \phi k_{\mu} k_{\nu}
$$

where $k_{\mu}$ is precisely the vector of eq. (3.2) and different functional forms for $\phi$ lead to different solutions. The scalar function corresponding to an accelerating particle is given by

$$
\phi=\frac{M}{4 \pi r} .
$$

Plugging this into the Einstein equations, one finds

$$
G_{\nu}^{\mu} \equiv R_{\nu}^{\mu}-\frac{R}{2} \delta^{\mu}{ }_{\nu}=\frac{\kappa^{2}}{2} T_{\mathrm{KS}}{ }^{\mu}{ }_{\nu}
$$

where $^{2}$

$$
T_{\mathrm{KS}}^{\mu \nu}=\left.\frac{3 M}{4 \pi} \frac{k \cdot \dot{\lambda}}{r^{2}} k^{\mu} k^{\nu}\right|_{\mathrm{ret}} .
$$

Thus, the use of Kerr-Schild coordinates for the accelerating particle leads to the presence of a non-trivial energy-momentum tensor on the right-hand side of the Einstein equations. We can already see that this extra term vanishes in the stationary case $\left(\dot{\lambda}^{\mu}=0\right)$, consistent with the results of ref. [55]. More generally, this stress-energy tensor $T_{\mathrm{KS}}^{\mu \nu}$ describes a pure radiation field present in the spacetime. The physical interpretation of this source is particularly clear in the electromagnetic "single copy" of this system, to which we now turn.

\section{$3.2 \quad$ Single copy}

Having examined a point particle in arbitrary motion in a Kerr-Schild spacetime, we may apply the classical single copy of eq. (2.5) to construct a corresponding gauge theoretic solution. This procedure is not guaranteed to work, given that the single copy of refs. [55, 56]

\footnotetext{
${ }^{2}$ We note what appears to be a typographical error in ref. [63], where the energy-momentum tensor contains an overall factor of 4 rather than 3. We have explicitly carried out the calculation leading to eq. (3.7), and found agreement with refs. [60-62].
} 
was only shown to apply in the case of stationary fields. However, we will see that we can indeed make sense of the single copy in the present context. Indeed, the physical interpretation of the stress-energy tensor $T_{\mathrm{KS}}^{\mu \nu}$ we encountered in the gravitational situation is illuminated by the single copy.

The essence of the Kerr-Schild double-copy is a relationship between gauge theoretic solutions $A^{\mu}=k^{\mu} \phi$ and Kerr-Schild metrics which is simply expressed as $k_{\mu} \rightarrow k_{\mu} k_{\nu}$. Thus, the single-copy of

$$
h^{\mu \nu}=-\frac{M \kappa}{2} \frac{1}{4 \pi r} k^{\mu} k^{\nu}
$$

is $^{3}$

$$
A^{\mu}=g \frac{1}{4 \pi r} k^{\mu}
$$

where $g$ is the coupling constant. ${ }^{4}$ Inserting this gauge field into the Yang-Mills equations, one finds that nonlinear terms vanish, leaving the Maxwell equations

$$
\partial^{\mu} F_{\mu \nu}=j_{\mathrm{KS} \nu},
$$

where

$$
F_{\mu \nu}=\partial_{\mu} A_{\nu}-\partial_{\nu} A_{\mu}
$$

is the usual electromagnetic field strength tensor.

A key result is that we find that the current density appearing in the Maxwell equations is given by

$$
j_{\mathrm{KS} \nu}=\left.2 \frac{g}{4 \pi} \frac{k \cdot \dot{\lambda}}{r^{2}} k_{\nu}\right|_{\text {ret }} .
$$

It is important to note that the current density $j_{\mathrm{KS}}$ is related to the energy-momentum tensor, eq. (3.8), we encountered in the gravitational case. Indeed the relationship between these sources is in accordance with the Kerr-Schild double copy: it involves a single factor of the Kerr-Schild vector $k^{\mu}$, with similar prefactors, up to numerical constants. We will return to this interesting fact in the following section.

The role of the Kerr-Schild current density $j_{\mathrm{KS}}$ can be understood by examining our single-copy gauge field, eq. (3.10), in more detail. Let us compute the electromagnetic field strength tensor of this system. Using the results (3.3) and (3.4), it is easy to check that

$$
F_{\mu \nu}=\partial_{\mu} A_{\nu}-\partial_{\nu} A_{\mu}=\frac{g}{4 \pi r^{2}}\left(k_{\mu} \lambda_{\nu}-\lambda_{\mu} k_{\nu}\right) .
$$

A first observation about this field strength tensor is that it falls off as $1 / r^{2}$ and does not depend on the acceleration of the particle. Therefore, it does not describe the radiation field

\footnotetext{
${ }^{3}$ In principle, one should include an arbitrary colour index on the field strength and current density. Given that the field equations are abelian, however, we ignore this. The resulting solution can be easily embedded in a non-abelian theory, as in refs. [55, 56]. Note that the abelian character of this theory also implies that we make the replacement $\frac{M \kappa}{2} \rightarrow g$ (cf. eq. (38) from ref. [55]).

${ }^{4}$ The relative sign between $h_{\mu \nu}$ and $A_{\mu}$ is necessary in our conventions to ensure that positive masses yield attractive gravitational fields while positive scalar potentials $A^{0}$ are sources for electric field lines $\boldsymbol{E}=-\nabla A^{0}$
} 
of the accelerated point particle in empty space, since the radiation fields must fall off as $1 / r$ and are linear in the acceleration. Secondly, this tensor is manifestly constructed from Lorentz covariant quantities. In the instantaneous rest frame of the particle, $\lambda^{\mu}=(1,0,0,0)$ and $k^{\mu}=(1, \hat{\boldsymbol{r}})$, and in this frame it is easy to see that the field strength is simply the Coulomb field of the point charge. Therefore, in a general inertial frame, our field strength tensor describes precisely the boosted Coulomb field of a point charge, omitting the radiation field completely.

The absence of radiation in the electromagnetic field strength makes the interpretation of the current density $j_{\mathrm{KS}}$ in the Maxwell equation obvious. This source must describe the radiation field of the point particle. To see this more concretely, let us compare our Kerr-Schild gauge field to the standard Liénard-Wiechert solution $A_{\mathrm{LW}}^{\mu}=\frac{g}{4 \pi r} \lambda^{\mu}$, which describes a point particle moving in an arbitrary manner in empty space (see e.g. [64]). This comparison is facilitated by defining a "radiative gauge field"

$$
A_{\mathrm{rad}}^{\mu}=\frac{g}{4 \pi r}\left(\lambda^{\mu}-k^{\mu}\right)
$$

which satisfies

$$
F_{\mathrm{rad}}^{\mu \nu} \equiv \partial^{\mu} A_{\mathrm{rad}}^{\nu}-\partial^{\nu} A_{\mathrm{rad}}^{\mu}=\frac{g}{4 \pi r}\left(k^{\mu} \beta^{\nu}-\beta^{\mu} k^{\nu}\right),
$$

where $\beta_{\mu}=\dot{\lambda}_{\mu}-\lambda_{\mu} k \cdot \dot{\lambda}$. Thus, $F_{\text {rad }}^{\mu \nu}$ is the radiative field strength of the point particle: it is linear in the particle acceleration, and falls off as $1 / r$ at large distances.

Now, since the Liénard-Wiechert field is a solution of the vacuum Maxwell equation, we know that $\partial_{\mu}\left(F^{\mu \nu}+F_{\text {rad }}^{\mu \nu}\right)=0$ and, consequently,

$$
\partial_{\mu} F_{\mathrm{rad}}^{\mu \nu}=-j_{\mathrm{KS}}^{\nu}
$$

We interpret $j_{\mathrm{KS}}$ as a divergence of the radiative field strength: we have put the radiation part of the gauge field on the right-hand side on the Maxwell equations, rather than the left.

Let us now summarise what has happened. By choosing Kerr-Schild coordinates for the accelerating particle in gravity, an extra energy-momentum tensor $T_{\mathrm{KS}}^{\mu \nu}$ appeared on the right-hand side of the Einstein equations. The single copy turns an energy density into a charge density (as in refs. $[55,56,59]$ ). Thus, the energy-momentum tensor in the gravity theory becomes a charge current $j_{\mathrm{KS}}^{\mu}$ in the gauge theory. We have now seen that this current represents the radiation coming from the accelerating charged particle, and this also allows us to interpret the corresponding energy-momentum tensor on the gravity side: it represents gravitational radiation from an accelerating point mass.

Indeed, our use of Kerr-Schild coordinates forced the radiation to appear in this form. The vector $k_{\mu}$ which is so crucial for our approach is twist-free: $\partial_{\mu} k_{\nu}=\partial_{\nu} k_{\mu}$. It is known that twist-free, vacuum, Kerr-Schild metrics are of Petrov type D, and therefore there is no gravitational radiation in the metric; see ref. [63] for a review. Correspondingly, the radiation is described by the Kerr-Schild sources.

The radiation fields of the accelerating charge in gauge theory, and the accelerating point mass in gravity, are described in Kerr-Schild coordinates by sources $j_{\mathrm{KS}}^{\mu}$ and $T_{\mathrm{KS}}^{\mu \nu}$. The structure of these sources reflects the Kerr-Schild double copy procedure: up to numerical 
factors, one replaces the vector $k_{\mu}$ by the symmetric trace-free tensor $k_{\mu} k_{\nu}$ to pass from gauge theory to gravity. This relationship between the sources, which describe radiation, is highly suggestive. Indeed, it is a standard fact that scattering amplitudes can be obtained from (amputated) currents. We may therefore anticipate that the structural relationship between the Kerr-Schild currents is related to the standard double copy for scattering amplitudes.

Nevertheless, there are still some puzzles regarding the analysis above. What, for example, are we to make of the different numerical factors appearing in the definitions eqs. (3.8) and (3.13) of the Kerr-Schild stress tensor and current density? If these sources are related to amplitudes, we expect a double copy which is local in momentum space. How can our currents be local in position space? More generally, how can we be sure that the Kerr-Schild double copy is indeed related to the standard BCJ procedure? The answer to these questions is addressed in the following section, in which we interpret the radiative sources directly in terms of scattering amplitudes.

Before proceeding, however, let us comment on the physical interpretation of the particle in the solutions under study. We considered how the particle affects the gauge or gravity fields, but we did not consider the cause of the acceleration of the particle, i.e. its own equation of motion. In the standard Liénard-Wiechert solution, the acceleration is due to a background field. It is therefore required that this background field does not interact with the radiation, otherwise the solution is not valid. This is true in electromagnetism or in its embedding in Yang-Mills theory. However, in the gravity case, one cannot envisage such a situation. Therefore, one should think of this particle merely as a boundary condition, and not as a physical particle subject to forces which would inevitably affect the Einstein equations. What we are describing here is a mathematical map between solutions in gauge theory and gravity, a map which exists irrespective of physical requirements on the solutions. In a similar vein, ref. [59] showed that energy-momentum tensors obtained through the classical double copy do not necessarily obey the positivity of energy conditions in general relativity.

\section{From Kerr-Schild sources to amplitudes}

In the previous section, we saw that the Kerr-Schild double copy can indeed describe radiating particles. The radiation appears as a source term on the right-hand side of the field equations. In this section, we consider a special case of this radiation, namely Bremsstrahlung associated with a sudden rapid change in direction. By Fourier transforming the source terms in the gauge and gravity theory to momentum space, we will see that they directly yield known scattering amplitudes which manifestly double copy. Moreover, the manipulations required to extract the scattering amplitudes in gauge theory and in gravity are precisely parallel. We will preserve the double copy structure at each step, so that the double copy property of the scattering amplitudes emerges from the $k_{\mu} \rightarrow k_{\mu} k_{\nu}$ structure of the Kerr-Schild double copy. In this way, we firmly establish a link between the classical double copy and the BCJ double copy of scattering amplitudes. 
In order to study Bremsstrahlung, we consider a particle which moves with velocity

$$
\lambda^{\mu}(\tau)=u^{\mu}+f(\tau)\left(u^{\prime \mu}-u^{\mu}\right)
$$

where

$$
f(\tau)= \begin{cases}0, & \tau<-\epsilon \\ 1, & \tau>\epsilon\end{cases}
$$

and, in the interval $(-\epsilon, \epsilon), f(\tau)$ is smooth but otherwise arbitrary. This describes a particle which moves with constant velocity $\lambda^{\mu}=u^{\mu}$ for $\tau<-\epsilon$, while for $\tau>\epsilon$ the particle moves with a different constant velocity $\lambda^{\mu}=u^{\prime \mu}$. Thus, the particle undergoes a rapid change of direction around $\tau=0$, assuming $\epsilon$ to be small. The form of $f(\tau)$ acts as a regulator needed to avoid pathologies in the calculation that follows. However, dependence on this regulator cancels out, so that an explicit form for $f(\tau)$ will not be needed. Owing to the constant nature of $u$ and $u^{\prime}$, the acceleration is given by

$$
\dot{\lambda}^{\mu}=\dot{f}(\tau)\left(u^{\prime \mu}-u^{\mu}\right) .
$$

The acceleration vanishes for $\tau<-\epsilon$ and $\tau>\epsilon$, but is potentially large in the interval $(-\epsilon, \epsilon)$. Without loss of generality, we may choose the spatial origin to be the place at which the particle changes direction, so that $y^{\mu}(0)=0$.

\subsection{Gauge theory}

We first consider the gauge theory case, and start by using the definitions of eqs. (3.2) to write the current density of eq. (3.13) as

$$
j_{\mathrm{KS}}^{\nu}=\frac{2 g}{4 \pi} \int d \tau \frac{\dot{\lambda}(\tau) \cdot(x-y(\tau))}{[\lambda(\tau) \cdot(x-y(\tau))]^{4}}(x-y(\tau))^{\nu} \delta\left(\tau-\tau_{\mathrm{ret}}\right)
$$

where we have introduced a delta function to impose the retarded time constraint. Using the identity

$$
\frac{\delta\left(\tau-\tau_{\text {ret }}\right)}{\lambda \cdot(x-y(\tau))}=2 \theta\left(x^{0}-y^{0}(\tau)\right) \delta\left((x-y(\tau))^{2}\right),
$$

one may rewrite eq. (4.4) as

$$
j_{\mathrm{KS}}^{\nu}=\frac{4 g}{4 \pi} \int d \tau \frac{\dot{\lambda}(\tau) \cdot(x-y(\tau))}{[\lambda(\tau) \cdot(x-y(\tau))]^{3}}(x-y(\tau))^{\nu} \theta\left(x^{0}-y^{0}(\tau)\right) \delta\left((x-y(\tau))^{2}\right) .
$$

Any radiation field will be associated with the non-zero acceleration only for $|\tau|<\epsilon$, where $y^{\mu}(\tau)$ is small. We may thus neglect this with respect to $x^{\mu}$ in eq. (4.6). Substituting eq. (4.3) then gives

$$
j_{\mathrm{KS}}^{\nu}=\frac{4 g}{4 \pi} x^{\nu} \theta\left(x^{0}\right) \delta\left(x^{2}\right) \int_{-\epsilon}^{\epsilon} d \tau \frac{b \dot{f}(\tau)}{(a+b f(\tau))^{3}}
$$

where

$$
a=x \cdot u, \quad b=x \cdot u^{\prime}-x \cdot u
$$


The integral is straightforwardly carried out to give

$$
\begin{aligned}
j_{\mathrm{KS}}^{\nu} & =-\frac{2 g}{4 \pi} x^{\nu} \theta\left(x^{0}\right) \delta\left(x^{2}\right)\left[\frac{1}{\left(x \cdot u^{\prime}\right)^{2}}-\frac{1}{(x \cdot u)^{2}}\right] \\
& =\frac{2 g}{4 \pi} \theta\left(x^{0}\right) \delta\left(x^{2}\right)\left[\frac{\partial}{\partial u_{\nu}^{\prime}}\left(\frac{1}{x \cdot u^{\prime}}\right)-\left(u^{\prime} \rightarrow u\right)\right] .
\end{aligned}
$$

One may now Fourier transform this expression, obtaining a current depending on a momentum $k$ conjugate to the position $x$. As our aim is to extract a scattering amplitude from the Fourier space current, $\tilde{j}_{\mathrm{KS}}^{\mu}(k)$, we consider only the on-shell limit of the current where $k^{2}=0$; we also drop terms in $\tilde{j}_{\mathrm{KS}}^{\mu}(k)$ which are proportional to $k^{\mu}$ as these terms are pure gauge. The technical details are presented in appendix A, and the result is

$$
\tilde{j}_{\mathrm{KS}}^{\nu}(k)=-i g\left(\frac{u^{\prime \nu}}{u^{\prime} \cdot k}-\frac{u^{\nu}}{u \cdot k}\right) .
$$

We may now interpret this as follows. First, we note that the current results upon acting on the radiative gauge field with an inverse propagator, consistent with the LSZ procedure for truncating Green's functions. It follows that the contraction of $\tilde{j}_{\mathrm{KS}}^{\nu}$ with a polarisation vector gives the scattering amplitude for emission of a gluon. Upon doing this, one obtains the standard eikonal scattering amplitude for Bremsstrahlung (see e.g. [65])

$$
\mathcal{A}_{\text {gauge }} \equiv \epsilon_{\nu}(k) \tilde{j}_{\mathrm{KS}}^{\nu}=-i g\left(\frac{\epsilon \cdot u^{\prime}}{u^{\prime} \cdot k}-\frac{\epsilon \cdot u}{u \cdot k}\right) .
$$

We thus see directly that the additional current density in the Kerr-Schild approach corresponds to the radiative part of the gauge field.

\subsection{Gravity}

We now turn to the gravitational case. Our goal is to extract the eikonal scattering amplitude for gravitational Bremsstrahlung from the Kerr-Schild stress-energy tensor $T_{\mathrm{KS}}^{\mu \nu}$ for a particle of mass $M$ moving along precisely the same trajectory as our point charge. Thus, the acceleration of the particle is, again,

$$
\dot{\lambda}^{\mu}=\dot{f}(\tau)\left(u^{\prime \mu}-u^{\mu}\right) .
$$

The calculation is a precise parallel to the calculation of the Bremsstrahlung amplitude for the point charge. However, as we will see, the presence of an additional factor of the Kerr-Schild vector $k^{\nu}$ in the gravitational case leads to a slightly different integral which we encounter during the calculation. This integral cancels the factor of 3 which appears in $T_{\mathrm{KS}}^{\mu \nu}$, restoring the expected numerical factors in the momentum space current. Let us now turn to the explicit calculation.

We begin by writing the stress tensor as an integral over a delta function which enforces the retardation and causality constraints

$$
T_{\mathrm{KS}}^{\mu \nu}=\frac{3 M}{2 \pi} \int d \tau \frac{\dot{\lambda}(\tau) \cdot(x-y(\tau))}{[\lambda(\tau) \cdot(x-y(\tau))]^{4}}(x-y(\tau))^{\mu}(x-y(\tau))^{\nu} \theta\left(x^{0}-y^{0}(\tau)\right) \delta\left((x-y(\tau))^{2}\right),
$$


corresponding to eq. (4.6) in the gauge theoretic case. The fourth power in the denominator in the gravitational case arises as a consequence of the additional factor of $k^{\mu}=(x-$ $y(\tau))^{\mu} /[\lambda(\tau) \cdot(x-y(\tau))]$. As before, the integral is strongly peaked around $y^{\mu}=0$, and we may perform the integral in this region to find that

$$
\begin{aligned}
T_{\mathrm{KS}}^{\mu \nu} & =-\frac{2 M}{4 \pi} x^{\mu} x^{\nu} \theta\left(x^{0}\right) \delta\left(x^{2}\right)\left[\frac{1}{\left(x \cdot u^{\prime}\right)^{3}}-\frac{1}{(x \cdot u)^{3}}\right] \\
& =-\frac{M}{4 \pi} \theta\left(x^{0}\right) \delta\left(x^{2}\right)\left[\frac{\partial}{\partial u_{\mu}^{\prime}} \frac{\partial}{\partial u_{\nu}^{\prime}}\left(\frac{1}{x \cdot u^{\prime}}\right)-\left(u^{\prime} \rightarrow u\right)\right] .
\end{aligned}
$$

Notice that the factor 3 in the numerator of the stress-energy tensor has cancelled due to the additional factor of $\lambda(\tau) \cdot(x-y(\tau))$ in the denominator of the integrand in the gravitational case. The double copy structure is evidently now captured by a replacement of one derivative $\frac{\partial}{\partial u_{\nu}^{\prime}}$ in gauge theory with two derivatives $\frac{\partial}{\partial u_{\mu}^{\prime}} \frac{\partial}{\partial u_{\nu}^{\prime}}$ in gravity.

Our next step is to Fourier transform to momentum space. The calculation is extremely similar to the gauge theoretic case (again, see appendix A). As our goal is to compute a scattering amplitude, we work in the on-shell limit $k^{2}=0$ and omit pure gauge terms. After a short calculation, we find

$$
\tilde{T}_{\mathrm{KS}}^{\mu \nu}(k)=-i M\left(\frac{u^{\prime \mu} u^{\prime \nu}}{u^{\prime} \cdot k}-\frac{u^{\mu} u^{\nu}}{u \cdot k}\right) .
$$

To construct the scattering amplitude, we must contract this Fourier-transformed stressenergy tensor with a polarisation tensor, which may be written as an outer product of two gauge theory polarisation vectors:

$$
\epsilon^{\mu \nu}(k)=\epsilon^{\mu}(k) \epsilon^{\nu}(k)
$$

The scattering amplitude is then given by

$$
\mathcal{A}_{\mathrm{grav}} \equiv \epsilon_{\mu}(k) \epsilon_{\nu}(k) \tilde{T}_{\mathrm{KS}}^{\mu \nu}(k)=-i M\left(\frac{\epsilon \cdot u^{\prime} \epsilon \cdot u^{\prime}}{u^{\prime} \cdot k}-\frac{\epsilon \cdot u \epsilon \cdot u}{u \cdot k}\right),
$$

corresponding to the known eikonal amplitude for gravitational Bremsstrahlung [66]. Again we see that the additional source term in the Kerr-Schild approach corresponds to the radiative part of the field. Furthermore, in this form the standard double copy for scattering amplitudes is manifest: numerical factors agree between eqs. (4.10) and (4.15), such that the mass in the gravity theory is replaced with the colour charge in the gauge theory, as expected from the usual operation of the classical single copy $[55,56]$.

Let us summarise the results of this section. We have examined the particular case of a particle which undergoes a rapid change in direction, and confirmed that the additional source terms appearing in the Kerr-Schild description (in both gauge and gravity theory) are exactly given by known radiative scattering amplitudes. This directly links the classical double copy to the BCJ procedure for amplitudes.

It is interesting to compare the BCJ double copy for scattering amplitudes with the Kerr-Schild double copy, which has been formulated in position space. It is clear that momentum space is the natural home of the double copy. For scattering amplitudes, the 
amplitudes themselves and the double copy procedure are local in momentum space. In our Bremsstrahlung calculation, the numerical coefficients in the sources are also more natural after the Fourier transform. On the other hand, the currents $T_{\mathrm{KS}}^{\mu \nu}$ and $j_{\mathrm{KS}}^{\nu}$ are also local in position space. This unusual situation arises because the scattering amplitudes do not conserve momentum: in any Bremsstrahlung process, some momentum must be injected in order to bend the point particle trajectory. Of course, in the case of a static point particle locality in both position space and momentum space is more natural. This is reflected by the structure of the Fourier transform in the present case: as explained in appendix A, the factor $1 / x \cdot u$ describing a particle worldline Fourier transforms to an integrated delta function $\int_{0}^{\infty} d m \delta^{4}(q-m u)$ (see eq. (A.3)).

\section{Gravitational energy conditions}

In this section, we consider the null, weak and strong energy conditions of general relativity. These were recently examined in the context of the Kerr-Schild double copy in ref. [59], where it was shown that extended charge distributions double copy to matter distributions that cannot simultaneously obey the weak and strong energy conditions, if there are no spacetime singularities or horizons. Although the point particle solution of interest to us has both singularities and horizons, it is still interesting to examine the energy conditions.

The null energy condition on a given energy-momentum tensor can be expressed by

$$
T_{\mu \nu} \ell^{\mu} \ell^{\nu} \geq 0
$$

where $\ell^{\mu}$ is any future-pointing null vector. The weak energy condition is similarly given by

$$
T_{\mu \nu} t^{\mu} t^{\nu} \geq 0
$$

for any future-pointing timelike vector $t^{\mu}$. The interpretation of this condition is that observers see a non-negative matter density. The null energy condition is implied by the weak energy condition (despite the names, the former is the weakest condition). One may also stipulate that the trace of the tidal tensor measured by such an observer is non-negative, which leads to the strong energy condition

$$
T_{\mu \nu} t^{\mu} t^{\nu} \geq \frac{T}{2} g_{\mu \nu} t^{\mu} t^{\nu}, \quad T \equiv T_{\alpha}^{\alpha}
$$

Let us now examine whether these conditions are satisfied by the Kerr-Schild energymomentum tensor of eq. (3.8). First, the null property of the vector $k^{\mu}$ implies that the trace vanishes, so that the weak and strong energy conditions are equivalent. We may further unify these with the null energy condition, by noting that eq. (3.8) implies

$$
T_{\mathrm{KS}}^{\mu \nu} V_{\mu} V_{\nu}=(k \cdot \dot{\lambda})\left[\frac{3 M(k \cdot V)^{2}}{4 \pi r^{2}}\right] .
$$

for any vector $V^{\mu}$. The quantity in the square brackets is positive definite, so that whether or not the energy conditions are satisfied is purely determined by the sign of $k \cdot \dot{\lambda}$. This 


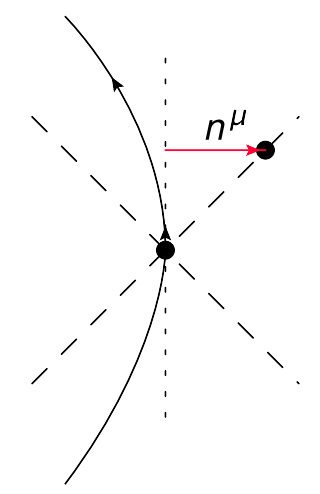

Figure 2. Physical interpretation of $(k \cdot \dot{\lambda})$, where this denotes the component of acceleration in the direction $n^{\mu}$.

scalar quantity is easily determined in the instantaneous rest-frame of the point particle; it is the negative of the component of acceleration in the direction $n^{\mu}$ of the observer (at the retarded time), see figure 2. Thus the energy conditions are not satisfied throughout the spacetime. In particular, any observer which sees the particle accelerating towards (away from) her will measure a negative (positive) energy density.

We remind the reader that the energy-momentum tensor is, in the case under study, an effective way of representing the full vacuum solution. The latter will have no issues with energy conditions. Analogously, the Liénard-Wiechert vacuum solution in gauge theory can be represented, as we have shown in section 3, by a boosted Coulomb field, together with a charged current encoding the radiation.

\section{Discussion}

In this paper, we have extended the classical double copy of refs. $[55,56]$ to consider accelerating, radiating point sources. This significantly develops previous results, which were based on stationary Kerr-Schild solutions, to a situation involving explicit time dependence. The structure of the double copy we have observed in the radiating case is precisely as one would expect. Passing from the gauge to the gravity theory, the overall scalar function $\phi$ is left intact; indeed it is the well-known scalar propagator in four dimensions. This is the same as the treatment of scalar propagators in the original BCJ double copy procedure for amplitudes. Similarly, the tensor structure of the gravitational field is obtained from the gauge field by replacing the vector $k_{\mu}$ by the symmetric, trace-free tensor $k_{\mu} k_{\nu}$. Finally, our use of Kerr-Schild coordinates in gravity linearised the Einstein tensor (with mixed indices). Reflecting this linearity, the associated single copy satisfies the linearised Yang-Mills equations.

It is worth dwelling a little on the physical implication of our work. The classical double copy is known to relate point sources in gauge theory to point sources in general relativity, in accordance with intuition arising from scattering amplitudes. In this article, we have simply considered the case where the point sources move on a specified, arbitrarily accelerated, timelike worldline. On general grounds we expect radiation to be emitted 
due to the acceleration. Our use of Kerr-Schild coordinates organised the radiation into sources appearing on the right-hand side of the field equations: a current density in gauge theory, and a stress-energy tensor in gravity. Intriguingly, we found that the expressions for these sources also have a double copy structure: one passes from the gauge current to the gravitational stress-energy tensor by replacing $k_{\mu}$ by $k_{\mu} k_{\nu}$ while leaving a scalar factor intact, up to numerical factors which are canonical in momentum space. Since these sources encode the complete radiation fields for the accelerating charge and black hole, there is a double copy between the radiation generated by these two systems. This double copy is a property of the exact solution of gauge theory and general relativity.

We further extracted one simple perturbative scattering amplitude from this radiation field, namely the Bremsstrahlung scattering amplitude. The double copy property was maintained as we extracted the scattering amplitude, which firmly establishes a link between the double copy for amplitudes and the double copy for classical solutions.

However, we should emphasise one unphysical aspect of our setup. We mandated a wordline for our point particle in both gauge theory and general relativity. In gauge theory, this is fine: one can imagine that an external force acts on the particle causing its worldline to bend. However, in general relativity such an external force would contribute to the stress-energy tensor in the spacetime. Since we ignored this component of the stressenergy tensor, our calculation is not completely physical. Instead, one should regard the point particle in both cases as a specified boundary condition, rather than as a physical particle. We have therefore seen that the radiation generated by this boundary condition enjoys a precise double copy.

There are a number of possible extensions of our results. One may look at timedependent extended sources in the Kerr-Schild description, for example, or particles accelerating in non-Minkowski backgrounds (for preliminary work in the stationary case, see ref. [56]). It would also be interesting to examine whether a double copy procedure can be set up in other coordinate systems, such as the more conventional de Donder gauge. One particularly important issue is to understand the generalisation of the colour-dual requirement on kinematic numerators to classical field backgrounds. The Jacobi relations satisfied by colour-dual numerators hint at the existence of a kinematic algebra [12,67] underlying the connection between gauge theory and gravity; revealing the full detail of this structure would clearly be an important breakthrough. The study of the classical double copy is in its infancy, and many interesting avenues have yet to be explored.

\section{Acknowledgments}

We thank John Joseph Carrasco and Radu Roiban for many illuminating discussions and thought-provoking questions. CDW is supported by the U.K. Science and Technology Facilities Council (STFC) under grant ST/L000446/1, and is perennially grateful to the Higgs Centre for Theoretical Physics for hospitality. DOC is supported in part by the STFC consolidated grant "Particle Physics at the Higgs Centre", by the National Science Foundation under grant NSF PHY11-25915, and by the Marie Curie FP7 grant 631370. AL is supported by Conacyt and SEP-DGRI studentships. 


\section{A Fourier transform of source terms}

In this appendix, we describe how to carry out the Fourier transform of eqs. (4.9), (4.14), to get the momentum-space expressions of eqs. (4.10), (4.15).

One may first consider the transform of $(u \cdot x)^{-1}$, where we work explicitly in four spacetime dimensions:

$$
\begin{aligned}
\mathcal{F}\left\{\frac{1}{u \cdot x}\right\} & =\int d^{4} x \frac{e^{i q \cdot x}}{u \cdot x} \\
& =\frac{1}{u^{0}} \int d^{3} x e^{-i \boldsymbol{q} \cdot \boldsymbol{x}} \int d x^{0} \frac{e^{i q^{0} x^{0}}}{x^{0}-\frac{\boldsymbol{x} \cdot \boldsymbol{u}}{u^{0}}} .
\end{aligned}
$$

Closing the $x^{0}$ contour in the upper half plane gives a positive frequency solution $q^{0}>0$ :

$$
\begin{aligned}
\mathcal{F}\left\{\frac{1}{u \cdot x}\right\} & =\frac{2 \pi i}{u^{0}} \int d^{3} x e^{-i \boldsymbol{x} \cdot\left[\boldsymbol{q}-\frac{q^{0}}{u^{0}} \boldsymbol{u}\right]} \\
& =\frac{i(2 \pi)^{4}}{u^{0}} \delta^{(3)}\left(\boldsymbol{q}-\frac{q^{0}}{u^{0}} \boldsymbol{u}\right) .
\end{aligned}
$$

It is possible to regain a covariant form for this expression by introducing a mass variable $m$, such that

$$
\begin{aligned}
\mathcal{F}\left\{\frac{1}{u \cdot x}\right\} & =\frac{i(2 \pi)^{4}}{u^{0}} \int_{0}^{\infty} d m \delta\left(m-\frac{q^{0}}{u^{0}}\right) \delta^{(3)}(\boldsymbol{q}-m \boldsymbol{u}) \\
& =i(2 \pi)^{4} \int_{0}^{\infty} d m \delta^{(4)}(q-m u),
\end{aligned}
$$

where the integral is over non-negative values of $m$ only, given that $q^{0}>0$. Given that $\theta\left(x^{0}\right) \delta\left(x^{2}\right)$ is a retarded propagator, ${ }^{5}$ one may also note the transform

$$
\mathcal{F}\left\{\theta\left(x^{0}\right) \delta\left(x^{2}\right)\right\}=-\frac{2 \pi}{q^{2}} .
$$

We then use the convolution theorem to obtain the Fourier transform of the current from eq. (4.9). The theorem states that the Fourier transform of a product is equal to the convolution of the transforms of each term. That is,

$$
\mathcal{F}\{f \cdot g\}=\mathcal{F}\{f\} * \mathcal{F}\{g\},
$$

where the convolution operation in four dimensions takes the form

$$
(F * G)(k)=\frac{1}{(2 \pi)^{4}} \int d^{4} q F(q) G(k-q) .
$$

Then, we can compute the Fourier transform of the current

$$
\begin{aligned}
\tilde{j}^{\nu}(k) & =\mathcal{F}\left\{j_{\mathrm{KS}}^{\nu}(x)\right\} \\
& =\frac{2 g}{4 \pi} \frac{\partial}{\partial u_{\nu}^{\prime}}\left[\mathcal{F}\left\{\theta\left(x^{0}\right) \delta\left(x^{2}\right)\right\} * \mathcal{F}\left\{\frac{1}{x \cdot u^{\prime}}\right\}\right]-\left(u \leftrightarrow u^{\prime}\right),
\end{aligned}
$$

\footnotetext{
${ }^{5}$ The retarded nature of the propagator is implemented by the prescription $\frac{1}{\left(p^{0}+i \varepsilon\right)^{2}-p^{2}}$, where $\varepsilon$ ensures convergence of the integrals in what follows.
} 
so inserting eqs. (A.4) and (A.3), and using the convolution definition eq. (A.6) we obtain the expression

$$
\begin{aligned}
\tilde{j}^{\nu}(k) & =\frac{2 g}{4 \pi} \frac{\partial}{\partial u_{\nu}^{\prime}}\left[\frac{1}{(2 \pi)^{4}} \int d^{4} q\left(-\frac{2 \pi}{q^{2}}\right)\left(i(2 \pi)^{4} \int_{0}^{\infty} d m \delta^{(4)}\left(k-q-m u^{\prime}\right)\right)\right]-\left(u \leftrightarrow u^{\prime}\right) \\
& =-i g \int_{0}^{\infty} d m\left(\frac{\partial}{\partial u_{\nu}^{\prime}}\left[\frac{1}{\left(k-m u^{\prime}\right)^{2}}\right]-\left(u \leftrightarrow u^{\prime}\right)\right) .
\end{aligned}
$$

where we have carried out the integral over $q$ in the last line. The derivative in the $m$ integral can be carried out to give

$$
\int_{0}^{\infty} d m \frac{2 m\left(k-m u^{\prime}\right)^{\nu}}{\left(k-m u^{\prime}\right)^{4}}=-\int_{0}^{\infty} d m \frac{2 m^{2} u^{\prime \nu}}{\left(m^{2}-2 m u^{\prime} \cdot k\right)^{2}}
$$

where, on the right-hand side, we have used the onshellness condition $k^{2}=0$, and also neglected terms $\sim k^{\mu}$, which vanish upon contraction of the current with a physical polarisation vector. The remaining integral over $m$ is easily carried out, and leads directly to the result of eq. (4.10).

Similar steps to those leading to eq. (A.8) can be used to rewrite eq. (4.14) in the form

$$
T_{\mathrm{KS}}^{\mu \nu}=\frac{i M}{2} \int_{0}^{\infty} d m\left(\frac{\partial}{\partial u_{\mu}^{\prime}} \frac{\partial}{\partial u_{\nu}^{\prime}}\left[\frac{1}{\left(k-m u^{\prime}\right)^{2}}\right]-\left(u \leftrightarrow u^{\prime}\right)\right) .
$$

Carrying out the double derivative gives

$$
\begin{aligned}
\frac{\partial}{\partial u_{\mu}^{\prime}} \frac{\partial}{\partial u_{\nu}^{\prime}}\left[\frac{1}{\left(k-m u^{\prime}\right)^{2}}\right] & =-\frac{2 m^{2} \eta^{\mu \nu}}{\left(m^{2}-2 m u^{\prime} \cdot k\right)^{4}}+\frac{8 m^{2}\left(k-m u^{\prime}\right)^{\mu}\left(k-m u^{\prime}\right)^{\nu}}{\left(m^{2}-2 m u^{\prime} \cdot k\right)^{3}} \\
& \simeq \frac{8 m^{4} u^{\prime \mu} u^{\prime \nu}}{\left(m^{2}-2 m u^{\prime} \cdot k\right)^{3}},
\end{aligned}
$$

where in the second line we have again used onshellness $\left(k^{2}=0\right)$, and ignored terms which vanish when contracted with the graviton polarisation tensor. Substituting eq. (A.11) into eq. (A.10), the $m$ integral is straightforward, and one obtains the result of eq. (4.15).

Open Access. This article is distributed under the terms of the Creative Commons Attribution License (CC-BY 4.0), which permits any use, distribution and reproduction in any medium, provided the original author(s) and source are credited.

\section{References}

[1] H. Kawai, D.C. Lewellen and S.H.H. Tye, A Relation Between Tree Amplitudes of Closed and Open Strings, Nucl. Phys. B 269 (1986) 1 [INSPIRE].

[2] Z. Bern and A.K. Grant, Perturbative gravity from QCD amplitudes, Phys. Lett. B 457 (1999) 23 [hep-th/9904026] [INSPIRE].

[3] Z. Bern, J.J.M. Carrasco and H. Johansson, New Relations for Gauge-Theory Amplitudes, Phys. Rev. D 78 (2008) 085011 [arXiv:0805.3993] [InSPIRE].

[4] Z. Bern, J.J.M. Carrasco and H. Johansson, Perturbative Quantum Gravity as a Double Copy of Gauge Theory, Phys. Rev. Lett. 105 (2010) 061602 [arXiv: 1004.0476] [INSPIRE]. 
[5] Z. Bern, T. Dennen, Y.-t. Huang and M. Kiermaier, Gravity as the Square of Gauge Theory, Phys. Rev. D 82 (2010) 065003 [arXiv: 1004.0693] [InSPIRE].

[6] N.E.J. Bjerrum-Bohr, P.H. Damgaard and P. Vanhove, Minimal Basis for Gauge Theory Amplitudes, Phys. Rev. Lett. 103 (2009) 161602 [arXiv:0907.1425] [INSPIRE].

[7] S. Stieberger, Open and Closed vs. Pure Open String Disk Amplitudes, arXiv:0907.2211 [INSPIRE].

[8] N.E.J. Bjerrum-Bohr, P.H. Damgaard, T. Sondergaard and P. Vanhove, Monodromy and Jacobi-like Relations for Color-Ordered Amplitudes, JHEP 06 (2010) 003 [arXiv:1003.2403] [INSPIRE].

[9] B. Feng, R. Huang and Y. Jia, Gauge Amplitude Identities by On-shell Recursion Relation in S-matrix Program, Phys. Lett. B 695 (2011) 350 [arXiv:1004.3417] [INSPIRE].

[10] S.H. Henry Tye and Y. Zhang, Dual Identities inside the Gluon and the Graviton Scattering Amplitudes, JHEP 06 (2010) 071 [Erratum ibid. 1104 (2011) 114] [arXiv:1003.1732] [INSPIRE].

[11] C.R. Mafra, O. Schlotterer and S. Stieberger, Explicit BCJ Numerators from Pure Spinors, JHEP 07 (2011) 092 [arXiv: 1104.5224] [INSPIRE].

[12] R. Monteiro and D. O'Connell, The Kinematic Algebra From the Self-Dual Sector, JHEP 07 (2011) 007 [arXiv: 1105.2565] [INSPIRE].

[13] N.E.J. Bjerrum-Bohr, P.H. Damgaard, R. Monteiro and D. O'Connell, Algebras for Amplitudes, JHEP 06 (2012) 061 [arXiv:1203.0944] [INSPIRE].

[14] Z. Bern, L.J. Dixon, D.C. Dunbar, M. Perelstein and J.S. Rozowsky, On the relationship between Yang-Mills theory and gravity and its implication for ultraviolet divergences, Nucl. Phys. B 530 (1998) 401 [hep-th/9802162] [INSPIRE].

[15] M.B. Green, J.H. Schwarz and L. Brink, $N=4$ Yang-Mills and $N=8$ Supergravity as Limits of String Theories, Nucl. Phys. B 198 (1982) 474 [INSPIRE].

[16] Z. Bern, J.S. Rozowsky and B. Yan, Two loop four gluon amplitudes in $N=4$ super Yang-Mills, Phys. Lett. B 401 (1997) 273 [hep-ph/9702424] [INSPIRE].

[17] S. Oxburgh and C.D. White, BCJ duality and the double copy in the soft limit, JHEP 02 (2013) 127 [arXiv:1210.1110] [INSPIRE].

[18] J.J. Carrasco and H. Johansson, Five-Point Amplitudes in $N=4$ super-Yang-Mills Theory and $N=8$ Supergravity, Phys. Rev. D 85 (2012) 025006 [arXiv:1106.4711] [INSPIRE].

[19] J.J.M. Carrasco, M. Chiodaroli, M. Günaydin and R. Roiban, One-loop four-point amplitudes in pure and matter-coupled $N \leq 4$ supergravity, JHEP 03 (2013) 056 [arXiv: 1212.1146] [INSPIRE].

[20] C.R. Mafra and O. Schlotterer, The Structure of n-Point One-Loop Open Superstring Amplitudes, JHEP 08 (2014) 099 [arXiv: 1203.6215] [INSPIRE].

[21] R.H. Boels, R.S. Isermann, R. Monteiro and D. O'Connell, Colour-Kinematics Duality for One-Loop Rational Amplitudes, JHEP 04 (2013) 107 [arXiv:1301.4165] [INSPIRE].

[22] N.E.J. Bjerrum-Bohr, T. Dennen, R. Monteiro and D. O'Connell, Integrand Oxidation and One-Loop Colour-Dual Numerators in N=4 Gauge Theory, JHEP 07 (2013) 092 [arXiv: 1303.2913] [INSPIRE]. 
[23] Z. Bern, S. Davies, T. Dennen, Y.-t. Huang and J. Nohle, Color-Kinematics Duality for Pure Yang-Mills and Gravity at One and Two Loops, Phys. Rev. D 92 (2015) 045041 [arXiv: 1303.6605] [INSPIRE].

[24] Z. Bern, S. Davies and T. Dennen, The Ultraviolet Structure of Half-Maximal Supergravity with Matter Multiplets at Two and Three Loops, Phys. Rev. D 88 (2013) 065007 [arXiv: 1305.4876] [INSPIRE].

[25] J. Nohle, Color-Kinematics Duality in One-Loop Four-Gluon Amplitudes with Matter, Phys. Rev. D 90 (2014) 025020 [arXiv:1309.7416] [INSPIRE].

[26] Z. Bern, S. Davies, T. Dennen, A.V. Smirnov and V.A. Smirnov, Ultraviolet Properties of $N=4$ Supergravity at Four Loops, Phys. Rev. Lett. 111 (2013) 231302 [arXiv:1309.2498] [INSPIRE].

[27] S.G. Naculich, H. Nastase and H.J. Schnitzer, All-loop infrared-divergent behavior of most-subleading-color gauge-theory amplitudes, JHEP 04 (2013) 114 [arXiv:1301.2234] [INSPIRE].

[28] Y.-J. Du, B. Feng and C.-H. Fu, Dual-color decompositions at one-loop level in Yang-Mills theory, JHEP 06 (2014) 157 [arXiv: 1402.6805] [INSPIRE].

[29] C.R. Mafra and O. Schlotterer, Towards one-loop SYM amplitudes from the pure spinor BRST cohomology, Fortsch. Phys. 63 (2015) 105 [arXiv:1410.0668] [INSPIRE].

[30] Z. Bern, S. Davies and T. Dennen, Enhanced ultraviolet cancellations in $\mathcal{N}=5$ supergravity at four loops, Phys. Rev. D 90 (2014) 105011 [arXiv: 1409.3089] [INSPIRE].

[31] C.R. Mafra and O. Schlotterer, Two-loop five-point amplitudes of super Yang-Mills and supergravity in pure spinor superspace, JHEP 10 (2015) 124 [arXiv: 1505.02746] [INSPIRE].

[32] S. He, R. Monteiro and O. Schlotterer, String-inspired BCJ numerators for one-loop MHV amplitudes, JHEP 01 (2016) 171 [arXiv:1507.06288] [INSPIRE].

[33] Z. Bern, S. Davies and J. Nohle, Double-Copy Constructions and Unitarity Cuts, Phys. Rev. D 93 (2016) 105015 [arXiv:1510.03448] [INSPIRE].

[34] G. Mogull and D. O'Connell, Overcoming Obstacles to Colour-Kinematics Duality at Two Loops, JHEP 12 (2015) 135 [arXiv:1511.06652] [INSPIRE].

[35] M. Chiodaroli, M. Günaydin, H. Johansson and R. Roiban, Spontaneously Broken Yang-Mills-Einstein Supergravities as Double Copies, arXiv:1511.01740 [INSPIRE].

[36] R. Saotome and R. Akhoury, Relationship Between Gravity and Gauge Scattering in the High Energy Limit, JHEP 01 (2013) 123 [arXiv:1210.8111] [INSPIRE].

[37] A. Sabio Vera, E. Serna Campillo and M.Á. Vázquez-Mozo, Color-Kinematics Duality and the Regge Limit of Inelastic Amplitudes, JHEP 04 (2013) 086 [arXiv:1212.5103] [INSPIRE].

[38] H. Johansson, A. Sabio Vera, E. Serna Campillo and M.Á. Vázquez-Mozo, Color-Kinematics Duality in Multi-Regge Kinematics and Dimensional Reduction, JHEP 10 (2013) 215 [arXiv: 1307.3106] [INSPIRE].

[39] H. Johansson, A. Sabio Vera, E. Serna Campillo and M.Á. Vázquez-Mozo, Color-kinematics duality and dimensional reduction for graviton emission in Regge limit, arXiv:1310.1680 [INSPIRE].

[40] R. Monteiro and D. O'Connell, The Kinematic Algebras from the Scattering Equations, JHEP 03 (2014) 110 [arXiv:1311.1151] [INSPIRE]. 
[41] M. Tolotti and S. Weinzierl, Construction of an effective Yang-Mills Lagrangian with manifest BCJ duality, JHEP 07 (2013) 111 [arXiv:1306.2975] [INSPIRE].

[42] C.-H. Fu, Y.-J. Du and B. Feng, Note on Construction of Dual-trace Factor in Yang-Mills Theory, JHEP 10 (2013) 069 [arXiv:1305.2996] [INSPIRE].

[43] Y.-J. Du, B. Feng and C.-H. Fu, The Construction of Dual-trace Factor in Yang-Mills Theory, JHEP 07 (2013) 057 [arXiv: 1304.2978] [INSPIRE].

[44] C.-H. Fu, Y.-J. Du and B. Feng, An algebraic approach to BCJ numerators, JHEP 03 (2013) 050 [arXiv: 1212.6168] [INSPIRE].

[45] S.G. Naculich, Scattering equations and BCJ relations for gauge and gravitational amplitudes with massive scalar particles, JHEP 09 (2014) 029 [arXiv: 1407.7836] [INSPIRE].

[46] S.G. Naculich, Scattering equations and virtuous kinematic numerators and dual-trace functions, JHEP 07 (2014) 143 [arXiv: 1404.7141] [INSPIRE].

[47] M. Chiodaroli, M. Günaydin, H. Johansson and R. Roiban, Scattering amplitudes in $\mathcal{N}=2$ Maxwell-Einstein and Yang-Mills/Einstein supergravity, JHEP 01 (2015) 081 [arXiv: 1408.0764] [INSPIRE].

[48] J.J.M. Carrasco, R. Kallosh, R. Roiban and A.A. Tseytlin, On the U(1) duality anomaly and the $S$-matrix of $N=4$ supergravity, JHEP 07 (2013) 029 [arXiv: 1303.6219] [INSPIRE].

[49] S. Litsey and J. Stankowicz, Kinematic numerators and a double-copy formula for $N=4$ super-Yang-Mills residues, Phys. Rev. D 90 (2014) 025013 [arXiv:1309.7681] [INSPIRE].

[50] S. Nagy, Chiral Squaring, arXiv:1412.4750 [INSPIRE].

[51] A. Anastasiou, L. Borsten, M.J. Hughes and S. Nagy, Global symmetries of Yang-Mills squared in various dimensions, JHEP 01 (2016) 148 [arXiv:1502.05359] [INSPIRE].

[52] H. Johansson and A. Ochirov, Color-Kinematics Duality for QCD Amplitudes, JHEP 01 (2016) 170 [arXiv:1507.00332] [INSPIRE].

[53] S. Lee, C.R. Mafra and O. Schlotterer, Non-linear gauge transformations in D $=10 S Y M$ theory and the BCJ duality, JHEP 03 (2016) 090 [arXiv:1510.08843] [INSPIRE].

[54] S.M. Barnett, Maxwellian theory of gravitational waves and their mechanical properties, New J. Phys. 16 (2014) 023027 [inSPIRE].

[55] R. Monteiro, D. O'Connell and C.D. White, Black holes and the double copy, JHEP 12 (2014) 056 [arXiv: 1410.0239] [INSPIRE].

[56] A. Luna, R. Monteiro, D. O'Connell and C.D. White, The classical double copy for Taub-UT spacetime, Phys. Lett. B 750 (2015) 272 [arXiv: 1507.01869] [INSPIRE].

[57] A.H. Taub, Empty space-times admitting a three parameter group of motions, Annals Math. 53 (1951) 472.

[58] E. Newman, L. Tamburino and T. Unti, Empty-pace generalization of the Schwarzschild metric, J. Math. Phys. 4 (1963) 915.

[59] A.K. Ridgway and M.B. Wise, Static Spherically Symmetric Kerr-Schild Metrics and Implications for the Classical Double Copy, arXiv:1512.02243 [INSPIRE].

[60] E.T. Newman and T.W.J. Unti, A class of null flat-space coordinate systems, J. Math. Phys. 4 (1963) 1467. 
[61] W. Kinnersley and M. Walker, Uniformly accelerating charged mass in general relativity, Phys. Rev. D 2 (1970) 1359 [InSPIRE].

[62] P. Vaidya and W. Bonnor, Exact solutions of the Einstein-Maxwell equations for an accelerated charge, (1972) [INSPIRE].

[63] H. Stephani, D. Kramer, M.A. MacCallum, C. Hoenselaers and E. Herlt, Exact solutions of Einstein's field equations, Cambridge University Press, Cambridge U.K. (2003).

[64] J.D. Jackson, Classical electrodynamics, third edition, Wiley, New York U.S.A. (1999).

[65] M.E. Peskin and D.V. Schroeder, An Introduction to quantum field theory, Westview Press, Boulder U.S.A. (1995).

[66] S. Weinberg, Infrared photons and gravitons, Phys. Rev. 140 (1965) B516.

[67] C.-H. Fu and K. Krasnov, Colour-Kinematics duality and the Drinfeld double of the Lie algebra of diffeomorphisms, arXiv:1603.02033 [INSPIRE]. 